Received: 31.08 .2018

Revised: 24.09.2018

Accepted: 19.10 .2018

DOI: $10.17804 / 2410-9908.2018 .5 .057-063$

\title{
A PROCEDURE FOR ONLINE INVESTIGATION OF DEUTERIUM DIFFUSION IN MATERIALS
}

\author{
O. A. Nefedova ${ }^{1,)^{*}}$ and V. B. Vykhodets ${ }^{2}$ \\ ${ }^{I}$ Institute of Engineering Science, Ural Branch of the Russian Academy of Sciences, \\ 34 Komsomolskaya St., Ekaterinburg, 620049, Russian Federation \\ ${ }^{2}$ M.N. Miheev Institute of Metal Physics, Ural Branch of the Russian Academy of Sciences, \\ 18 S. Kovalevskoy St., Ekaterinburg, 620108, Russian Federation \\ https://orcid.org/0000-0003-1163-6699, , nefedova@imach.uran.ru; \\ *Corresponding author. E-mail: nefedova@imach.uran.ru \\ Address for correspondence: ul. Komsomolskaya, 34, Ekaterinburg, 620049, Russian Federation \\ Tel.: +7 (343) 362-30-22
}

The work summarizes the application of online nuclear reaction analysis to the study of deuterium diffusion in materials. Two types of the internal diffusion source formed by the mechanism of ion implantation are considered. In one case, a deuterium solid solution is formed in the sample during implantation; in the other case, deuteride is formed. The formulation of the diffusion boundary value problem and the analytical solution are written for both cases. Simulation calculations are carried out and a criterion is constructed for identifying the diffusion source type and choosing an adequate scheme of experimental data processing. The application of the criterion in the study of deuterium diffusion in nickel and sodium samples is described.

Keywords: deuterium diffusion, internal source, deuterium solid solution, deuteride, nuclear reaction analysis, diffusion boundary value problem, analytical solution.

\section{Acknowledgements}

The work was performed within the state assignment from FASO Russia (theme Spin, No. 01201463330) and partially supported by RFBR (project No. 17-03-00101).

\section{References}

1. Messer R., Blessing A., Dais S., Hupfel D., Majer G., Schmidt C., Seeger A., Zag W. Nuclear Magnetic Resonance Studies of Hydrogen Diffusion, Trapping, and Site Occupation in Metals. Zeitschrift $f_{b r}$ Physikalische Chemie Neue Folge, 1986, vol. 2, pp. 61-119. DOI: https://doi.org/10.1524/zpch.1986.1986.Suppl_2.061.

2. $\quad$ Qi Zh., Vцlkl J., Lдsser R., Wenzl H. Tritium diffusion in V, Nb and Ta. Journal of Physics F: Metal Physics, 1983, vol. 13, num. 10, pp. 2053-2062. DOI: https://doi.org/10.1088/03054608/13/10/015.

3. $\quad$ Vykhodets V.B., Kurennykh T.E., Nefedova O.A., Gorelov V.P., Stroeva A.Yu., Balakireva V.B., Vykhodets E.V., Obukhov S.I. Deuterium diffusion in proton conductors $\mathrm{La}_{0.9} \mathrm{Sr}_{0.1} \mathrm{ScO}_{3-\delta}$ and $\mathrm{BaZr}_{0.9} \mathrm{Y}_{0.1} \mathrm{O}_{3-\delta}$ at room temperature. Solid State Ionics, 2014, vol. 263, pp. 152-156. DOI: $10.1016 /$ j.ssi.2014.06.003.

4. Vykhodets V.B., Kurennykh T.E., Nefedova O.A. Application of Nuclear Reaction Analysis to Studying Hydrogen Diffusion and Nonstoichiometry in Metal Oxides. Diagnostics, Resource and Mechanics of materials and structures, 2015, iss. 5, pp. 31-44. Available at: http://dreamjournal.org/issues/2015-5/2015-5 57.html 
5. Vykhodets V.B., Kurennykh T.E., Gorelov V.P., Balakireva V.B., Vykhodets E.V., Obukhov S.I. Hydrogen isotope exchange in proton-conducting oxides during proton and deuteron irradiation. JETP Letters, 2013, vol. 97, iss. 11, pp 624-627. DOI: https://doi.org/10.1134/S0021364013110106.

6. Kartashov E.M. Analiticheskie metody v teorii teploprovodnosti tverdykh tel [Analytical Methods in the Theory of Heat Conduction of Solids]. M., Vysshaya Shkola Publ., 2001, 550 p. (In Russian).

7. Crank J. The mathematics of diffusion. London, Oxford University Press Publ., 1975, 414 p. ISBN 0-19-853344-6.

8. Carslaw H.S., Jaeger J.C. Conduction of Heat in Solids. London, Oxford University Press Publ., 1959, 510 p. ISBN-10: 0198533039, ISBN-13: 978-0198533030.

9. SRIM-The Stopping and Ranges of Ions in Matter. Available at: http://www.srim.org. 
Подана в журнал: 31.08 .2018

УДК 544.6:544.16:517.958

DOI: $10.17804 / 2410-9908.2018 .5 .057-063$

\title{
МЕТОДИКА ДЛЯ ИССЛЕДОВАНИЯ ДИФФУЗИИ ДЕЙТЕРИЯ В МАТЕРИАЛАХ В РЕЖИМЕ ОН-ЛАЙН
}

\author{
О. А. Нефедова ${ }^{1, \text { a)* }}$, В. Б. Выходец ${ }^{2}$ \\ ${ }^{1}$ Федеральное государственное бюджетное учреждение науки \\ Институт машиноведения Уральского отделения Российской академии наук, \\ ул. Комсомольская, 34, Екатеринбург, Российская Федерация \\ ${ }^{2}$ Федеральное государственное бюджетное учреждение науки \\ Институт физики металлов им. М.Н.Михеева Уральского отделения Российской академии наук, \\ ул. С. Ковалевской, 18, Екатеринбург, Российская Федераџия \\ a) iD https://orcid.org/0000-0003-1163-6699, nefedova@imach.uran.ru; \\ *Ответственный автор. Электронная почта: nefedova@imach.uran.ru \\ Адрес для переписки: ул. Комсомольская, 34, Екатеринбург, Российская Федерация \\ Тел.: +7 (343) 362-30-22
}

В работе выполнено обобщение применения ускорительной методики ядерных реакций в режиме он-лайн для исследования диффузии дейтерия в материалах. Рассмотрены два типа внутреннего источника диффузии, сформированного по механизму ионной имплантации. В первом случае во время имплантации в образце образуется твердый раствор дейтерия, а во втором случае - дейтерид. Для обоих случаев записаны постановка краевой задачи диффузии и соответствующее аналитическое решение. Проведены модельные расчеты и построен критерий для идентификации типа диффузионного источника и выбора адекватной схемы обработки опытных данных. Описано применение критерия при исследовании диффузии дейтерия в образцах никеля и натрия.

Ключевые слова: диффузия дейтерия, внутренний источник, твердый раствор дейтерия, дейтерид, ядерный микроанализ, краевая задача диффузии, аналитическое решение.

\section{1. Введение}

Данные по диффузии изотопов водорода в металлах представляют большой интерес для теории диффузии, атомной и водородной энергетики, материаловедения, катализа и многих других отраслей науки и техники. Это связано с наибольшей среди всех элементов скоростью диффузии водорода в твердых телах, с его участием во многих природных и технологических процессах, с важной ролью квантовых эффектов при диффузии изотопов водорода, с наличием у водорода трех изотопов с сильно различающимися массами, что является базой для получения данных по изотопным эффектам при диффузии. Особенностью работ по диффузии водорода в металлах является наличие большого числа экспериментальных методик. В их число входят прямые методы определения коэффициентов диффузии $D$, основанные на измерении зависимости концентрации атомов $c(x, t)$ от координаты $x$ в образце и времени отжига $t$, а также непрямые методы, например, ядерный магнитный резонанс, квазистатический и динамический эффекты Горского, магнитное последействие, электросопротивление, квазиупругое рассеяние нейтронов, Мессбауэровская спектроскопия и т. д. Несмотря на это в литературе полностью отсутствуют опытные данные по диффузии изотопов водорода в щелочных металлах в большинстве щелочноземельных, в металлах с низкой растворимостью водорода, почти во всех металлах при криогенных температурах. Связано это с тем, что такие металлы являются неудобными объектами для исследования диффузии водорода, в них, например, сложно ввести атомы водорода или, напротив, избежать образование гидридов и т. д. 
В работе [1] для исследования диффузии одного из изотопов водорода - дейтерия - была предложена ускорительная методика ядерных реакций в режиме он-лайн - NRAOL. При еe использовании образец размещается в вакуумной камере ускорительной установки, его температура поддерживается постоянной и осуществляется непрерывное облучение образца дейтронами. При облучении происходит формирование внутреннего источника диффузии атомов дейтерия в образце по механизму ионной имплантации, диффузия атомов дейтерия из этого источника к поверхности и вглубь образца, и в режиме он-лайн проводятся измерения концентрационного профиля $c(x, t)$ с помощью ядерной реакции ${ }^{2} \mathrm{H}(\mathrm{d}, \mathrm{p})^{3} \mathrm{H}$. C помощью методики NRAOL измерения коэффициентов диффузии дейтерия в принципе могут быть выполнены для любых материалов при любых температурах, при которых возможен отжиг образцов в высоком вакууме. В работе [1] принималось, что при ионной имплантации образуются только твердые растворы дейтерия в образце, однако при ионной имплантации могут образоваться также дейтериды металла. Цель настоящей работы - разработка методики NRAOL для общего случая.

\section{2. Краевая задача диффузии для методики NRAOL}

Рассмотрим краевую задачу диффузии дейтерия для полуограниченного образца $0<x<\infty$, внутри которого непрерывно действует источник диффузии. Предположим, что при имплантации на глубине $x=x_{0}$ могут быть сформированы два типа внутреннего источника диффузии: 1 - твердый раствор дейтерия в образце, 2 - дейтерид. В первом случае диффузия дейтерия будет осуществляться из бесконечно тонкого слоя к облучаемой и необлучаемой поверхностям образца, при этом концентрация дейтерия на глубине $x=x_{0}$ будет возрастать со временем $t$ облучения образца. Во втором случае концентрация дейтерия ${ }^{*}$ на глубине $x=x_{0}$ не будет зависеть от времени $t$, она будет равна концентрации дейтерия в твердом растворе, находящемся в равновесии с дейтеридом. В общем случае концентрация ${ }^{*}{ }^{*}$ является функцией температуры образца. Можно ожидать, что реализация того или иного типа внутреннего источника диффузии будет зависеть от материала образца, его температуры и плотности тока пучка ускорителя. При втором типе диффузионного источника атомы дейтерия диффундируют в обоих направлениях от слоя дейтерида к облучаемой и необлучаемой поверхности образца. Отметим, что в рамках методики NRAOL информация о профилях $c(x, t)$ для обоих типов диффузионного источника может быть получена только для глубин $x<x_{0}$.

Запишем постановку краевой задачи для источника диффузии первого типа. В этом случае диффузия протекает из бесконечно тонкого слоя, расположенного на глубине $x=x_{0}$, и действие источника постоянной мощности $w$, помещенного на глубину $x=x_{0}$, моделирует функция $f(x, t)=$ $=w \delta\left(x-x_{0}\right)$, где $\delta\left(x-x_{0}\right)-$ дельта-функция Дирака. Если принять, что радиационные дефекты не влияют на коэффициенты диффузии, т.е. в облучаемой и необлучаемой зонах коэффициенты диффузии одинаковы и равны $D$, то уравнение, описывающее процесс диффузии, имеет вид:

$$
\frac{\partial c(x, t)}{\partial t}=D \frac{\partial^{2} c(x, t)}{\partial x^{2}}+f(x, t), \quad t>0, \quad 0<x<\infty .
$$

Используем следующие краевые условия задачи:

$$
c(x, 0)=0 ;\left.\frac{\partial c(x, t)}{\partial x}\right|_{x=0}=0, c(\infty, t)=0 .
$$

Они предполагают отсутствие атомов дейтерия в образце до облучения и нулевой поток атомов дейтерия через облучаемую поверхность образца.

Краевая задача (1), (2) была решена в работе [1] с применением подхода [2], при котором непрерывно действующий источник диффузии имитировался серией мгновенных источников, а распределение $c(x, t)$, соответствующее непрерывно действующему источнику, рассматривалось как суперпозиция решений, соответствующих мгновенным источникам. В результате было получено аналитическое выражение для распределения концентрации дейтерия в образце: 


$$
\begin{aligned}
c(x, t) & =\frac{w}{2 D}\left(\left(x-x_{0}\right)\left(\operatorname{erf}\left(\frac{x-x_{0}}{2 \sqrt{D t}}\right)-\operatorname{sgn}\left(x-x_{0}\right)\right)+\left(x+x_{0}\right)\left(\operatorname{erf}\left(\frac{x+x_{0}}{2 \sqrt{D t}}\right)-1\right)\right)+ \\
& +w \sqrt{\frac{t}{D \pi}}\left(\exp \left(\frac{x x_{0}}{D t}\right)+1\right) \exp \left(-\frac{\left(x+x_{0}\right)^{2}}{4 D t}\right) ; \operatorname{sgn}\left(x-x_{0}\right)=\left\{\begin{array}{c}
1, x>x_{0}, \\
-1, x<x_{0} .
\end{array}\right.
\end{aligned}
$$

Для источника второго типа задача сводится к решению дифференциального уравнения диффузии в ограниченном слое $0<x<x_{0}$

$$
\frac{\partial c(x, t)}{\partial t}=D \frac{\partial^{2} c(x, t)}{\partial x^{2}}, t>0,0<x<x_{0}
$$

Дополним задачу (4) краевыми условиями

$$
c(x, 0)=0 ;\left.\frac{\partial c(x, t)}{\partial x}\right|_{x=0}=0, c\left(x_{0}, t\right)=c^{*} .
$$

Будем искать решение с помощью метода разделения переменных (метод Фурье) [3]. Поскольку применение этого метода требует однородных граничных условий, то сделаем в уравнениях (4), (5) замену переменной $u(x, t)=c(x, t)-c^{*}$ и перейдем к вспомогательной задаче

$$
\frac{\partial u(x, t)}{\partial t}=D \frac{\partial^{2} u(x, t)}{\partial x^{2}}, t>0,0<x<x_{0}
$$

со следующими краевыми условиями:

$$
u(x, 0)=-c^{*} ;\left.\frac{\partial u(x, t)}{\partial x}\right|_{x=0}=0, u\left(x_{0}, t\right)=0
$$

Применим к решению (4a), (5a) метод разделения переменных и найдем решение вспомогательной задачи в виде ряда:

$$
u(x, t)=\frac{4 c^{*}}{\pi} \sum_{n=1}^{\infty} \frac{(-1)^{n}}{2 n-1} \exp \left(-\frac{D \pi^{2}(2 n-1)^{2}}{4 l^{2}} t\right) \cos \frac{\pi(2 n-1) x}{2 l} .
$$

С учетом проведенной выше замены переменных запишем решение исходной задачи (4) и (5):

$$
c(x, t)=c^{*}\left(1+\frac{4}{\pi} \sum_{n=1}^{\infty} \frac{(-1)^{n}}{2 n-1} \exp \left(-\frac{D \pi^{2}(2 n-1)^{2}}{4 l^{2}} t\right) \cos \frac{\pi(2 n-1) x}{2 l}\right) .
$$

Таким образом, в общем случае, применяя методику NRAOL, следует принимать во внимание существование двух выражений (3) и (6) для определения коэффициентов диффузии дейтерия, и актуальной задачей является разработка критерия для выбора адекватного алгоритма. Для получения информации по этому вопросу выполним модельные расчеты с помощью выражений (3) и (6). При их проведении были использованы следующие значения параметров: $x_{0}=14,85 \mathrm{M \kappa м} ; x=12,1 \mathrm{мкм} ; c^{*}=5 \cdot 10^{25} \mathrm{aT} / \mathrm{m}^{3} ; D_{1}=10^{-12} \mathrm{M}^{2} / \mathrm{c} ; D_{2}=10^{-13} \mathrm{M}^{2} / \mathrm{c}$; $D_{3}=10^{-14} \mathrm{M}^{2} / \mathrm{c}$. Они соответствовали применению методики NRAOL для исследования диффузии дейтерия в натрии при энергии ускоренных дейтронов 650 кэв. Для других металлов должны быть использованы другие характерные значения соответствующих параметров. 


\section{3. Результаты и обсуждение}

На рис. 1 представлены графики, характеризующие рассчитанные по формуле (3) временные зависимости концентрации дейтерия на глубине $x=12,1$ мкм для трех значений коэффициента диффузии $D_{1}>D_{2}>D_{3}$.

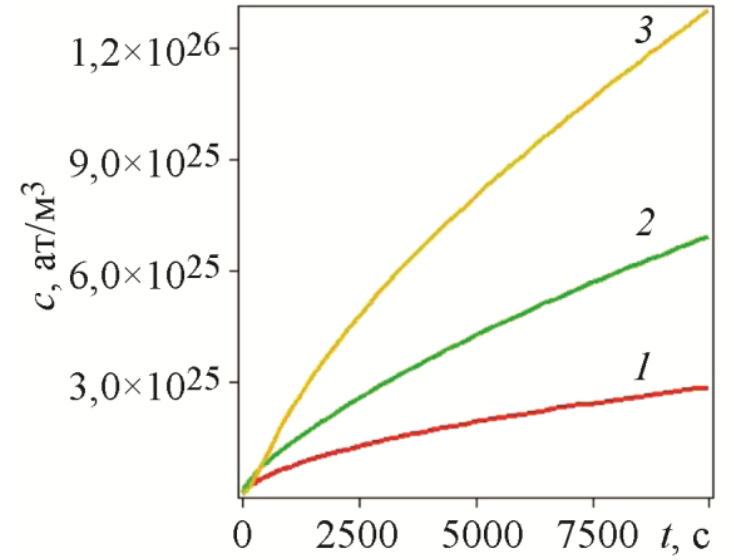

Рис. 1. Изменение концентрации дейтерия во времени на глубине 12,1 мкм для аналитического решения (3).

Кривые 1,2 и 3 соответствуют коэффициентам диффузии: $1-D_{1}=10^{-12} \mathrm{~m}^{2} / \mathrm{c}$, $2-D_{2}=10^{-13} \mathrm{M}^{2} / \mathrm{c}, 3-D_{3}=10^{-14} \mathrm{~m}^{2} / \mathrm{c}$

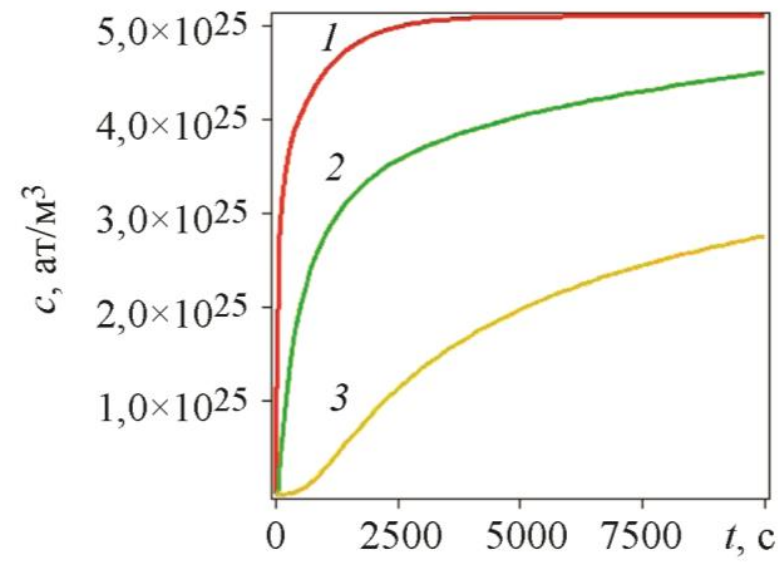

Рис. 2. Изменение концентрации дейтерия во времени на глубине $x=12,1$ мкм для аналитического решения (6).

Кривые 1,2 и 3 соответствуют коэффициентам диффузии: $1-D_{1}=10^{-12} \mathrm{M}^{2} / \mathrm{c}$, $2-D_{2}=10^{-13} \mathrm{M}^{2} / \mathrm{c}, 3-D_{3}=10^{-14} \mathrm{M}^{2} / \mathrm{c}$

Для сравнения, на рис. 2 приведены расчетные временные зависимости концентрации дейтерия на глубине $x=12,1$ мкм для $D_{1}, D_{2}, D_{3}$, построенные с помощью аналитического решения (6).

Из рис. 1 и 2 видно, что кривые, характеризующие изменение концентрации дейтерия во времени, при увеличении коэффициента диффузии ведут себя различным образом в зависимости от типа диффузионного источника. Для источника первого типа, когда при имплантации на глубине $x=x_{0}$ в образце образуется твердый раствор дейтерия в материале, более высоким значениям коэффициента диффузии для одних и тех же моментов времени будут соответствовать меньшие значения концентрации дейтерия. Из рис. 1 видно, что это будет иметь место при больших временах облучения образца. Для источника второго типа, когда при имплантации на глубине $x=x_{0}$ в образце образуется дейтерид, напротив, более высоким значениям коэффициентов диффузии будут соответствовать более высокие значения концентрации дейтерия. Это будет иметь место при любых временах облучения образцов.

С учетом результатов этих модельных расчетов может быть сформулирован критерий для идентификации типа диффузионного источника и, как следствие, осуществлен выбор выражения $c(x, t)$, которое должно быть использовано при математической обработке опытных данных. Из рис. 1 и 2 следует, что для идентификации типа диффузионного источника измерения зависимости $c(t)$ должны быть выполнены при близких значениях силы тока пучка для двух температур $T_{1}$ и $T_{2}$, которые не сильно отличаются друг от друга. Если будет установлено, что для одних и тех же моментов времени при $T_{1}<T_{2}$ выполняется соотношение $c\left(T_{1}\right)<c\left(T_{2}\right)$, то в диапазоне температур $T_{1}$ и $T_{2}$ функционирует источник диффузии второго типа, при котором во время имплантации на глубине $x=x_{0}$ в образце образуется дейтерид. Напротив, если $T_{1}<T_{2}$, но выполняется противоположное соотношение $c\left(T_{1}\right)>c\left(T_{2}\right)$, то в диапазоне температур $T_{1}$ и $T_{2}$ функционирует источник первого типа, при котором во время имплантации на глубине $x=x_{0}$ в образце образуется твердый раствор дейтерия в металле.

Этот критерий был апробирован при применении методики NRAOL для обработки опытных данных при исследовании диффузии дейтерия в никеле и натрии. Было установлено, 
что в никеле при всех температурах функционировал источник диффузии, при котором во время имплантации формировались твердые растворы дейтерия в металле. В натрии имела место более сложная ситуация: при очень низких температурах $T<-70{ }^{\circ} \mathrm{C}$ функционировал источник, при котором на глубине $x=x_{0}$ в образце формировался дейтерид, а при температурах $T>-50{ }^{\circ} \mathrm{C}-$ твердый раствор дейтерия в натрии.

\section{4. Заключение}

Разработан обобщенный вариант методики ядерных реакций в режиме он-лайн (NRAOL) для исследования диффузии дейтерия в материалах и сформулирован критерий для обработки опытных данных. Предметом анализа в работе был выбор краевых условий диффузионной задачи при обработке опытных диффузионных данных, рассмотрены два типа внутреннего источника диффузии, сформированного при имплантации ионов дейтерия в образцы: твердый раствор дейтерия и дейтерид. Было установлено, что достаточным критерием для выбора адекватной схемы обработки опытных данных является разность концентраций дейтерия на определенной глубине в образце при двух различных температурах образца. Ее положительное или отрицательное значение однозначно соответствует одному из двух типов источников диффузии. Результаты выполненного исследования подтвердили, что с помощью методики NRAOL измерения коэффициентов диффузии дейтерия могут быть выполнены в металлах, в которых дейтерий практически нерастворим и которые разрушаются при отжигах в водороде. Единственным ограничением при применении методики NRAOL является возможность проведения диффузионных отжигов образцов в высоком вакууме.

\section{Благодарность}

Работа выполнена в рамках государственного задания ФАСО России (тема «Спин» № 01201463330) и частично поддержана РФФИ (проект № 17-03-00101).

\section{Литература}

1. Nuclear Magnetic Resonance Studies of Hydrogen Diffusion, Trapping, and Site Occupation in Metals / R. Messer, A. Blessing, S. Dais, D. Hцpfel, G. Majer, C. Schmidt, A. Seeger, W. Zag // Zeitschrift fbr Physikalische Chemie Neue Folge. - 1986. - Vol. 2. - P. 61-119. DOI: https://doi.org/10.1524/zpch.1986.1986.Suppl_2.061.

2. Tritium diffusion in V, Nb and Ta / Zh. Qi, J. Vulkl, R. Lдsser, H. Wenzl // Journal of Physics F: Metal Physics - 1983. - Vol. 13, num. 10 - P. 2053-2062. DOI: https://doi.org/10.1088/0305-4608/13/10/015.

3. Deuterium diffusion in proton conductors $\mathrm{La}_{0.9} \mathrm{Sr}_{0.1} \mathrm{ScO}_{3-д}$ and $\mathrm{BaZr}_{0.9} \mathrm{Y}_{0.1} \mathrm{O}_{3-д}$ at room temperature / V. B. Vykhodets, T. E. Kurennykh, O. A. Nefedova, V. P. Gorelov, A. Yu. Stroeva, V. B. Balakireva, E. V. Vykhodets, S. I. Obukhov // Solid State Ionics. - 2014. - Vol. 263. P. 152-156. - DOI: 10.1016/j.ssi.2014.06.003.

4. $\quad$ Vykhodets V. B., Kurennykh T. E., Nefedova O. A. Application of Nuclear Reaction Analysis to Studying Hydrogen Diffusion and Nonstoichiometry in Metal Oxides // Diagnostics, Resource and Mechanics of materials and structures. - 2015. - Iss. 5. - P. 31-44. - URL: http://dreamjournal.org/issues/2015-5/2015-5_57.html

5. Hydrogen isotope exchange in proton-conducting oxides during proton and deuteron irradiation / V. B. Vykhodets, T. E. Kurennykh, V. P. Gorelov, V. B. Balakireva, E. V. Vykhodets, S. I. Obukhov // JETP Letters. - 2013. - Vol. 97, iss. 11. - P. 624-627. DOI: https://doi.org/10.1134/S0021364013110106.

6. Карташов Э. М. Аналитические методы в теории теплопроводности твердых тел. М. : Высш. шк., 2001.- 550 с. - ISBN 5-06-004091-7.

7. Crank J. The Mathematics of Diffusion. - London : Oxford University Press, 1975. 414 p. - ISBN 0-19-853344-6.

8. Carslaw H. S., Jaeger J. C. Conduction of Heat in Solids. - London : Oxford University Press, 1959. - 510 p. - ISBN-10: 0198533039, ISBN-13: 978-0198533030.

9. SRIM-The Stopping and Ranges of Ions in Matter [Электронный pecypc]. URL: http://www.srim.org (дата обращения: 02.04.2018). 